\title{
Heat Transfer Characteristics of Mixed Electroosmotic and Pressure Driven Micro-Flows*
}

\author{
Keisuke HORIUCHI** and Prashanta DUTTA***
}

\begin{abstract}
We analyze heat transfer characteristics of steady electroosmotic flows with an arbitrary pressure gradient in two-dimensional straight microchannels considering the effects of Joule heating in electroosmotic pumping. Both the temperature distribution and local Nusselt number are mathematically derived in this study. The thermal analysis takes into consideration of the interaction among advective, diffusive, and Joule heating terms to obtain the thermally developing behavior. Unlike macro-scale pipes, axial conduction in micro-scale cannot be negligible, and the governing energy equation is not separable. Thus, a method that considers an extended Graetz problem is introduced. Analytical results show that the Nusselt number of pure electrooosmotic flow is higher than that of plane Poiseulle flow. Moreover, when the electroosmotic flow and pressure driven flow coexist, it is found that adverse pressure gradient to the electroosmotic flow makes the thermal entrance length smaller and the heat transfer ability stronger than pure electroosmotic flow case.
\end{abstract}

Key Words: Mixed Electroosmotic and Pressure Driven Flows, Microfluidics, Joule Heating, Extended Graetz Problem

\section{Introduction}

The emerging lab-on-a-chip micro-fluidic devices are getting more attention in medical, pharmaceutical, and defense applications due to their low-cost, less operation time, light-weight, and small-size advantages. One of the key functionalities of these lab-on-a-chip devices is to transport liquid or reagent from one place to another. A recent study shows electroosmotic pumping is applicable to working fluids of a wide range of conductivity unlike others, and it can develop higher head capacity than other $\operatorname{methods}^{(1)}$.

Since the discovery of the electrokinetic phenomenon by F.F. Reuss in $1809^{(2)}$, there have been numerous analytical, experimental, and numerical studies on this phenomenon. However, so far, most of the studies have been focused on the fluid flow behavior under steady ${ }^{(3)-(5)}$ and unsteady ${ }^{(6),(7)}$ electric fields with uniform wall electrochemical conditions, and not much attention has been paid

${ }^{*}$ Received 14th December, 2005 (No. 05-4275)

** Hitachi Ltd., Mechanical Engineering Research Laboratory, 832-2 Horiguchi, Hitachinaka, Ibaraki 312-0034, Japan. E-mail: keisuke.horiuchi.fy@ hitachi.com

*** School of Mechanical and Materials Engineering, Washington State University, Pullman, WA 99164-2920, U.S.A. to the thermal behavior.

Because the electroosmotic flow is driven in the presence of a large external electric field, the interaction between the electric field and the charged ions results in a thermal energy generation as Joule heating. In typical electroosmotic flows with a fluid that has an electrical conductivity of $300 \mu \mathrm{S} / \mathrm{m}$ and an applied electric field of $100 \mathrm{~V} / \mathrm{mm}^{(8)}$, the Joule heating contributes $3 \times 10^{6} \mathrm{~W} / \mathrm{m}^{3}$. Recently Maynes and Webb have presented thermal behaviors of fully developed combined pressure and electroosmotically driven micro-flows ${ }^{(9)}$. However, their analysis is valid only in the fully developed region.

Analysis of energy equation in the developing region is particularly challenging for low Reynolds number flows ( $R e \leq 100)$, primarily due to the presence of axial conduction. This particular problem is known as an extended Graetz problem, where the associated eigenvalue problem is non-self-adjoint by referring to the standard SturmLiouville problem. In literature, none of the previous studies associated with Graetz problem have, until now, dealt with electroosmotic flow and/or volumetric Joule heating.

For this article, we obtained analytical solutions for the temperature distributions and heat transfer characteristics of mixed electroosmotic and pressure driven flows in two-dimensional microchannels. Both developing and fully developed regions have been analyzed for isothermal 
channel walls. We have utilized the separation of variables technique for homogeneous energy equations introduced by Lahjomri and Oubarra, where they solved the thermal problem for arbitrary velocity distribution without any heat generation ${ }^{(10)}$. Our analysis takes care of the interaction among advective, viscous, and Joule heating terms to obtain the temperature distribution within the fluid. This analysis especially identifies the effects of Joule heating and pressure gradient in microchannels during mixed electroosmotic and pressure driven pumping used for designing "lab-on-a-chip" micro-fluidic devices.

\section{Nomenclature}

$c_{p}:$ specific heat at constant pressure $[\mathrm{J} /(\mathrm{kg} \mathrm{K})]$

$D$ : half channel height [m]

$E_{x}$ : streamwise component of electric field [V/m]

${ }_{1} F_{1}$ : Kummer confluent hypergeometric function [-]

$G$ : normalized heat generation term,

$G \equiv \sigma(\vec{E} \cdot \vec{E}) D^{2} /\left\{k\left(T_{e}-T_{s}\right)\right\}[-]$

$h_{\xi}$ : local heat transfer coefficient $\left[\mathrm{W} /\left(\mathrm{m}^{2} \mathrm{~K}\right)\right]$

$k:$ thermal conductivity $[\mathrm{W} /(\mathrm{m} \mathrm{K})]$

$N u_{\xi}$ : local Nusselt number, $D h_{\xi} / k[-]$

$P$ : pressure $[\mathrm{Pa}]$

$P e_{T}$ : thermal Peclet number based on the electroosmotic velocity $[-]$

$R_{e v}$ : ratio of Joule heating to viscous dissipation [-]

$T:$ absolute temperature [K]

$U$ : non-dimensional streamwise velocity, $u / u_{H S}[-]$

$u:$ streamwise velocity component $[\mathrm{m} / \mathrm{s}]$

$u_{H S}$ : Helmholtz-Smoluchowski velocity, $-\varsigma \varepsilon E_{x} / \mu$ $[\mathrm{m} / \mathrm{s}]$

$\mathrm{x}:$ streamwise coordinate $[\mathrm{m}]$

$\mathrm{y}$ : cross flow coordinate $[\mathrm{m}]$

\section{Greek Letters}

$\varepsilon$ : permittivity of the medium $[\mathrm{C} /(\mathrm{V} \mathrm{m})]$

$\Phi$ : viscous dissipation $\left[\mathrm{W} / \mathrm{m}^{3}\right]$

$\eta$ : non-dimensional cross flow coordinate, $\eta=y / D$ $[-]$

$\lambda_{n}: n$-th eigenvalues [-]

$\mu:$ dynamic viscosity $[\mathrm{Pa} \mathrm{s}]$

$v$ : kinematic viscosity $\left[\mathrm{m}^{2} / \mathrm{s}\right]$

$\theta:$ non-dimensional temperature, $\theta \equiv\left(T-T_{S}\right) /\left(T_{e}-\right.$ $\left.T_{S}\right)[-]$

$\rho_{f}$ : fluid density $\left[\mathrm{kg} / \mathrm{m}^{3}\right]$

$\sigma$ : electrical conductivity of the buffer fluid $[\mathrm{S} / \mathrm{m}]$

$\Omega$ : non-dimensional pressure gradient, $\Omega \equiv-D^{2} /$ $\left(2 \mu u_{H S}\right) d P / d x[-]$

$\xi$ : non-dimensional streamwise coordinate, $\xi=x / D$ $[-]$

\section{Subscripts}

$\zeta$ : zeta potential [V]

$\mathrm{c}:$ value at the center $(y=0)$

$\mathrm{e}:$ value at the inlet $(x=0)$

$\mathrm{g}:$ general solution

$$
\begin{aligned}
& \mathrm{p}: \text { particular solution } \\
& \mathrm{s}: \text { value at the surface }(y=D)
\end{aligned}
$$

\section{Mathematical Model}

\subsection{Mixed electroosmotic and pressure driven flows}

Analysis of the electroosmotic flow in a twodimensional microchannel has been presented in a number of studies. The formation of an electric double layer (EDL) on a channel wall is required to have electroosmotic flows. The thickness of an EDL depends on the ion concentrations. This thickness of EDL typically varies from 1 to $10 \mathrm{~nm}$ corresponding to the 100 to $1 \mathrm{mM}$ buffer concentration. When the thickness of EDL thickness is very thin, the electroosmotic velocity is approximated as HelmholtzSmoluchowski velocity, $u_{H S}{ }^{(11),(12)}$, which is a function of the zeta potential, $\varsigma$, the permittivity of the medium, $\varepsilon$, the viscosity of the fluid, $\mu$, and the applied electric field in the streamwise direction, $E_{x}$. Although the electrokinetic flow is desired in many bioanalytical applications, there may be a pressure variation along the channel due to the following reasons:

- presence of an alternative pumping mechanism,

- placement of a mechanical valve in the flow path,

- head difference between the inlet and exit reservoirs.

The resulting pressure gradient distorts the plug-like velocity distribution (Fig. 1). In the absence of an inertial term due to a low Reynolds number $(R e)$, the momentum equation becomes linear. Therefore, the steady velocity distribution for the mixed electroosmotic and pres-

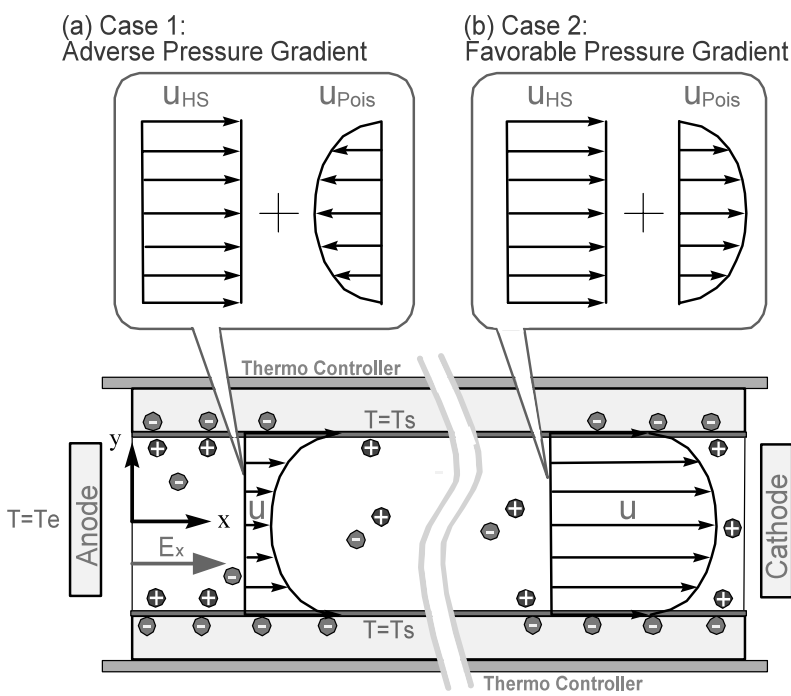

Fig. 1 Schematic of mixed electroosmotic and pressure driven flows: (a) adverse pressure gradient and (b) favorable pressure gradient. Under the ideal conditions of uniform zeta potential and electrolyte concentration, we can obtain pure electroosmotically driven plug flows if there is no pressure gradient 
sure driven flow in a two-dimensional straight channel can be obtained by considering the linear superposition of the electroosmotic velocity $\left(u_{H S}\right)$ and the plane Poiseulle flow velocity $\left(u_{P o i s}\right)^{(5)}$ as

$$
u(x, y)=u_{H S}+u_{\text {Pois }}=-\frac{\varsigma \varepsilon E_{x}}{\mu}-\frac{D^{2}}{2 \mu} \frac{d P}{d x}\left\{1-\left(\frac{y}{D}\right)^{2}\right\}
$$

where $P$ is the pressure and $D$ is the half channel height.

\subsection{Energy transport in mixed electroosmotic- pressure driven flows}

The governing equation for the two-dimensional steady thermal energy transport can be presented as

$$
\rho_{f} c_{P} u \frac{\partial T}{\partial x}=\frac{\partial T}{\partial x}\left(k \frac{\partial T}{\partial x}\right)+\frac{\partial T}{\partial y}\left(k \frac{\partial T}{\partial y}\right)+\Phi+\sigma E_{x}^{2},
$$

where $\rho_{f}$ is the fluid density, $c_{P}$ is the specific heat, $T$ is the absolute temperature, $k$ is the thermal conductivity, $\Phi \approx \mu(\partial u / \partial y)^{2}$ is the viscous dissipation, and $\sigma$ is the electrical conductivity of the buffer fluid. The last term, the specific term for electroosmotic flows, is called Joule heating simply because of the existence of an electric field. Since we have modeled the electrokinetic component of the flow velocity with the Helmholtz-Smoluchowski slip velocity, the viscous dissipation term is mainly due to the pressure driven component of the velocity. Here we assume that the flow is hydraulically fully developed from the very beginning of the channel, but the temperature field is developing. This can be justified for fluid whose Reynolds number is less than one (typical in microfluidics) and the Prandtl number is greater than one (such as de-ionized water with a $P r=7$ ), respectively. Therefore, for the hydraulically fully developed mixed electroosmotic and pressure driven flow in a two-dimensional straight channel, the ratio of Joule heating to the viscous dissipation can be expressed as,

$$
R_{e v}=\frac{\int_{-D}^{+D} \sigma E_{x}^{2} d y}{\int_{-D}^{+D} \mu(y / \mu d P / d x)^{2} d y} \approx \frac{3}{4} \mu \sigma\left(\frac{D}{\Omega \varepsilon \varsigma}\right)^{2},
$$

where $\Omega$ is the normalized pressure gradient $(\Omega \equiv$ $\left.-1 / 2 d P^{*} / d \xi\right)$ and $P^{*}$ is the non-dimensional pressure $\left(P^{*} \equiv P D /\left(\mu u_{H S}\right)\right)$. From Eq. (3), it is clear that the ratio of the generation terms, $R_{e v}$, is independent of the externally applied electric field, but depends on the characteristic dimensions, pressure gradient, zeta potential, and electric conductivity. For the flow and electrokinetic parameter ranges considered for this study, the same as in our previous work, the above ratio varies between 10 and 10000 . Therefore, we neglected the viscous dissipation term for this analysis.

For the isothermal surface case, the two-dimensional energy equation is subjected to the following boundary conditions.

$$
\begin{aligned}
& T=T_{e} \text { at } x=0,0 \leq y \leq D \\
& T<\infty \text { at } x \rightarrow \infty, 0 \leq y \leq D \\
& \frac{\partial T}{\partial y}=0 \text { at } 0 \leq x<\infty, y=0 \\
& T=T_{S} \text { at } 0 \leq x<\infty, y=D
\end{aligned}
$$

\subsection{Normalization scheme}

We normalize streamwise and cross-stream coordinates $(x, y)$ with the half channel height $D(\xi=x / D$, $\eta=y / D)$. Here, $D$ is used as the characteristic length as opposed to the hydraulic diameter, $D_{H}=4 D$. Therefore, the flow Reynolds and Nusselt numbers in this study will be one fourth of their conventional values, where those numbers are calculated based on hydraulic diameters. For convenience, we normalize the flow velocity with the Helmholtz-Smoluchowski velocity, $U=u / u_{H S}$. Under this normalization scheme, the non-dimensional streamwise velocity is

$$
U=u / u_{H S}=1+\Omega\left(1-\eta^{2}\right),
$$

where $\Omega$ is the non-dimensional pressure gradient. Knowing the inlet temperature, $T_{e}$, and the surface temperature, $T_{S}$, we now define the normalized temperature as $\theta \equiv\left(T-T_{S}\right) /\left(T_{e}-T_{S}\right)$ and the normalized heat generation term as $G \equiv \sigma(\vec{E} \cdot \vec{E}) D^{2} /\left\{k\left(T_{e}-T_{s}\right)\right\}$. Based on the above normalization scheme, the energy equation and corresponding boundary conditions can be written as follows:

$$
\begin{aligned}
& P e_{T} U \frac{\partial \theta}{\partial \xi}=\frac{\partial^{2} \theta}{\partial \xi^{2}}+\frac{\partial^{2} \theta}{\partial \eta^{2}}+G, \\
& \theta=1 \text { at } \xi=0,0 \leq \eta \leq 1, \\
& \theta<\infty \text { at } \xi \rightarrow \infty, 0 \leq \eta \leq 1, \\
& \frac{\partial \theta}{\partial \eta}=0 \text { at } 0 \leq \xi<\infty, \eta=0, \text { and } \\
& \theta=0 \text { at } 0 \leq \xi<\infty, \eta=1,
\end{aligned}
$$

where $P e_{T}$ is the thermal Peclet number for pure electroosmotic flow, and it can be found by multiplying the flow Reynolds number based on Helmholtz-Smoluchowski velocity with the fluid Prandtl number as $P e_{T}=u_{H S} D / \alpha$, where $\alpha$ is the thermal diffusivity. The normalized source term, $G$, represents a ratio of the energy generation to the thermal conduction. An aqueous solution (with a TrisEDTA buffer) of conductivity, $\sigma=90 \mu \mathrm{S} / \mathrm{cm}^{(7)}$, and an applied electric field of $400 \mathrm{~V} / \mathrm{mm}$, will contribute to $G=1$ in a $100-\mu \mathrm{m}$ thick microchannel for $T_{e}-T_{S}=5 \mathrm{~K}$. In the above normalization process, the temperature independence of the fluid properties is assumed. Although the fluid viscosity, $\mu$, and thermal conductivity, $k$, are strong functions of the fluid temperature, their variations can be neglected for a temperature difference of $10 \mathrm{~K}$ or smaller. Moreover, both the electric field, $\vec{E}=\left(E_{x}, 0\right)$, and the electrical conductivity, $\sigma$, are assumed uniform throughout the channel. The normalized energy equation presented by Eq. (6.a) is a second order partial differential equation. 


\section{Analysis of Normalized Energy Equation}

Let's decompose the normalized temperature, $\theta$, into two parts,

$$
\theta=\theta_{P}+\theta_{g}
$$

where $\theta_{P}$ is chosen so that $\theta_{g}$ satisfies a homogeneous equation. Now, we seek a particular solution $\theta_{P}$ as a function of an $\eta$ subject to the boundary conditions, Eqs. (6.d) and (6.e). We obtain a solution for a particular type of function:

$$
\theta_{P}=\frac{G}{2}\left(1-\eta^{2}\right)
$$

On the other hand, the governing equation for $\theta_{g}$ becomes homogeneous. Suppose the solution to the homogeneous problem has the following form (for details see Ref. (11)):

$$
\theta_{g}=\sum_{n=1}^{\infty} A_{n} f_{n}(\eta) \exp \left[-\frac{\lambda_{n}^{2}}{P e_{T}} \xi\right]
$$

where $A_{n}$ are the coefficients, $f_{n}$ are the eigenfunctions, and $\lambda_{n}$ are the eigenvalues. By substituting Eqs. (7) and (9) into Eq. (6), we obtain the following nonlinear eigenvalue problem:

$$
\begin{aligned}
& \frac{d^{2} f_{n}}{d \eta^{2}}+\lambda_{n}^{2}\left\{\left(\frac{\lambda_{n}}{P e_{T}}\right)^{2}+U\right\} f_{n}=0, \\
& \frac{d f_{n}}{d \eta}=0 \text { at } \eta=0, \text { and } \\
& f_{n}=0 \text { at } \eta=1
\end{aligned}
$$

The solution to Eq. (10.a) under a symmetric boundary condition, Eq. (10.b), can be expressed as

$$
f_{n}=\exp \left[-\frac{1}{2} \lambda_{n} \sqrt{\Omega} \eta^{2}\right]{ }_{1} F_{1}(a ; b ; z),
$$

where ${ }_{1} F_{1}(a ; b ; z)=\sum_{j=0}^{\infty}\left((a)_{j} /(b)_{j}\right)\left(z^{j} / j !\right)$ is the Kummer confluent hypergeometric function, $(a)_{j}$ and $(b)_{j}$ are known as the Pochhammer symbols, and the variable of the hypergeometric function in this particular study is

$$
\begin{aligned}
& a=\frac{-\lambda_{n}^{3}-P e_{T}^{2}\left(\lambda_{n}(1+\Omega)-\sqrt{\Omega}\right)}{4 \sqrt{\Omega} P e_{T}^{2}}, \\
& b=1 / 2, \text { and } \\
& z=\lambda_{n} \eta^{2} \sqrt{\Omega} .
\end{aligned}
$$

The eigenfunctions $f_{n}$ presented by Eq. (11.a) have a sequence of eigenvalues $\left\{\lambda_{n}\right\}$, and $\left\{f_{n}\right\}_{n=1}^{\infty}$ forms a base for the function space $\mathrm{L}^{2}(0,1)$. Our next goal is to find the eigenvalues $\left\{\lambda_{n}\right\}$ from Eq. (11.a) by utilizing the wall boundary condition given in Eq. (10.c). For this nonlinear eigenvalue problem, the Secant method is utilized to find out the corresponding eigenvalues, and they are presented in Table 1. Note that the eigenfunctions $\left\{f_{n}\right\}$ are not mutually orthogonal (by referring to the standard SturmLiouville problem) since the eigenvalues $\left\{\lambda_{n}\right\}$ occur nonlinearly in Eq. (10.a). We use the Gram-Schmidt orthogonal procedure in order to determine the coefficients, $\left\{A_{n}\right\}$,
Table 1 First ten terms of coefficients $\left(A_{n}\right)$ and corresponding eigenvalues $\left(\lambda_{n}\right)$ for $P e_{T}=1$

\begin{tabular}{|c|c|c|c|c|}
\cline { 2 - 5 } \multicolumn{1}{c|}{} & \multicolumn{3}{|c|}{$\Omega= \pm 1$} & \multicolumn{2}{c|}{$\Omega=0$} \\
\hline$n$ & $\lambda_{n}$ & $A_{n}$ & $\lambda_{n}$ & $A_{n}$ \\
\hline 1 & 0.945 & $+1.3 \times 10^{0}-5.2 \times 10^{-1} \mathrm{G}$ & 1.072 & $+1.3 \times 10^{0}-5.2 \times 10^{-1} \mathrm{G}$ \\
\hline 2 & 1.986 & $-4.2 \times 10^{-1}+2.2 \times 10^{-2} \mathrm{G}$ & 2.059 & $-4.2 \times 10^{-1}+2.0 \times 10^{-2} \mathrm{G}$ \\
\hline 3 & 2.657 & $+2.5 \times 10^{-1}-4.5 \times 10^{-3} \mathrm{G}$ & 2.715 & $+2.6 \times 10^{-1}-4.1 \times 10^{-3} \mathrm{G}$ \\
\hline 4 & 3.193 & $-1.8 \times 10^{-1}+1.6 \times 10^{-3} \mathrm{G}$ & 3.241 & $-1.8 \times 10^{-1}+1.5 \times 10^{-3} \mathrm{G}$ \\
\hline 5 & 3.651 & $+1.4 \times 10^{-1}-7.4 \times 10^{-4} \mathrm{G}$ & 3.694 & $+1.4 \times 10^{-1}-7.1 \times 10^{-4} \mathrm{G}$ \\
\hline 6 & 4.058 & $-1.2 \times 10^{-1}+4.0 \times 10^{-4} \mathrm{G}$ & 4.097 & $-1.2 \times 10^{-1}+3.9 \times 10^{-4} \mathrm{G}$ \\
\hline 7 & 4.428 & $+9.8 \times 10^{-2}-2.4 \times 10^{-4} \mathrm{G}$ & 4.464 & $+9.8 \times 10^{-2}-2.4 \times 10^{-4} \mathrm{G}$ \\
\hline 8 & 4.769 & $-8.5 \times 10^{-2}+1.6 \times 10^{-4} \mathrm{G}$ & 4.803 & $-8.5 \times 10^{-2}+1.5 \times 10^{-4} \mathrm{G}$ \\
\hline 9 & 5.088 & $+7.5 \times 10^{-2}-1.1 \times 10^{-4} \mathrm{G}$ & 5.119 & $+7.5 \times 10^{-2}-1.1 \times 10^{-4} \mathrm{G}$ \\
\hline 10 & 5.387 & $-6.7 \times 10^{-2}+7.7 \times 10^{-5} \mathrm{G}$ & 5.418 & $-6.7 \times 10^{-2}+7.5 \times 10^{-5} \mathrm{G}$ \\
\hline
\end{tabular}

from a set of linearly independent eigenfunctions $\left\{f_{n}\right\}_{n=1}^{\infty}$. Therefore, the normalized temperature distribution can be obtained from Eqs. (7) - (9) as

$$
\theta=\theta_{p}+\theta_{g}=\frac{G}{2}\left(1-\eta^{2}\right)+\sum_{n=1}^{\infty} A_{n} f_{n} \exp \left[-\frac{\lambda_{n}^{2}}{P e_{T}} \xi\right] .
$$

It is important to note that the normalized temperature distribution obtained in Eq. (12) is valid for $\Omega>-1.5$. If the normalized pressure gradient were less than -1.5 , the net flow would change direction, which is undesirable for the presented mathematical model. The corresponding Nusselt number for the mixed electroosmotic and pressure driven flow can be obtained by normalizing the heat transfer coefficient based on the Newton's Law of Cooling $\left(h_{\xi}=(-k \partial T / \partial y)_{y=D} /\left(T_{s}-T_{m}\right)=(k / D)(-\partial \theta / \partial \eta)_{\eta=1} / \theta_{m}\right)$.

$$
\begin{aligned}
N u_{\xi}= & \frac{D h_{\xi}}{k} \\
= & \frac{5(3+2 \Omega)\left(G-\left.\sum_{n=1}^{\infty} A_{n} \exp \left[-\frac{\lambda_{n}^{2}}{P e_{T}} \xi\right] \frac{\partial f_{n}}{\partial \eta}\right|_{\eta=1}\right)}{G(5+4 \Omega)+15 \sum_{n=1}^{\infty} A_{n} \exp \left[-\frac{\lambda_{n}^{2}}{P e_{T}} \xi\right] \int_{0}^{1} U f_{n} d \eta} .
\end{aligned}
$$

\section{Discussion of Results}

For mixed electroosmotic and pressure driven flows, the analytical solutions of the heat transfer characteristics are obtained with Eqs. (12) and (13). The convergence of the infinite series is very slow at $\xi=0$, and therefore, we have plotted our results from $\xi=0.01$. The analytical solutions presented here are for $\operatorname{Re}<1, \operatorname{Pr}>1$, and $D \geq 1000 \lambda$ where $\lambda$ is the Debye length. Therefore, the hydrodynamic entry length is negligible, and the mixed velocity distribution is justified from the very beginning of the channel. On the other hand, the thermally developing region can exist while hydraulically fully developed. 
In this section, we plot the local fluid temperature $(\theta)$ and Nusselt number $\left(N u_{\xi}\right)$ distribution along the channel. We particularly focus on the thermally developing behavior at the entry region, by considering the effects of Joule heating. Since Joule heating is a volumetric phenomenon, its effect is very significant in regular scale electrokinetic devices $^{(13)-(15)}$. We have presented the temperature distribution for only two extreme cases $(G=-1$ and $G=+1)$, considering practical fluid and thermal parameters. Here negative value of $G$ means that the inlet temperature $\left(T_{e}\right)$ is lower than the wall temperature $\left(T_{s}\right)$. The results demonstrated in this section are a representative for de-ionized water at $R e<0.7, P r=7$, and $P e_{T}<5$. Due to the symmetric boundary condition, the results are presented for only the upper half of the channel.

It is possible to find out the temperature distribution for any value of $\Omega>-1.5$. Here specific cases of $\Omega=0$, 1 , and -1 are discussed in Figs. $2-4$, respectively in order to see the significance of the favorable pressure gradient $(\Omega>0)$ and adverse pressure gradient $(\Omega<0)$. The normalized pressure gradient indicates a ratio of pressure driven velocity component at the channel centerline to the Helmholtz-Smoluchowski velocity. Therefore, the physical meaning of $|\Omega|=1$ is that the magnitude of the pressure driven velocity component at the centerline is the same as the Helmholtz-Smoluchowski velocity. The negative value of $\Omega$ represents the adverse pressure gradient flow, where there is a net gain in the pressure head. This adverse pressure gradient $(\Omega<0)$ normally happens more often compare to other cases $(\Omega \geq 0)$ because electroosmotic pumping creates a pressure head. Note that the negative value of $\theta$ happens if and only if the non-dimensional source term, $G$, is negative. This indicates that the dimensional temperature at the center, $T_{C}$, eventually becomes greater than the wall temperature, $T_{S}$, due to Joule heating even if the inlet temperature, $T_{e}$, is lower than the wall temperature, $T_{S}$.

The non-dimensional temperature distribution of mixed flow $(\Omega \neq 0)$ presented in Figs. 3 and 4 follows the very similar trend observed in the pure electroosmotic flow $(\Omega=0)$ in Fig. 2, and the temperature profile eventually reaches identical values in all cases as long as the magnitude of normalized source term $(G)$ is the same. It is noted that the developing speed varies significantly with the increase of the pressure gradient. For example, the non-dimensional temperature at $\xi=1$ and $\eta=0$ for $G=-1$ reaches $\theta_{C}=0.06,0.22$, and -0.11 for $\Omega=0,+1$, and -1 , respectively. For a particular generation term $(G)$, the longer thermal entrance length of mixed flows with favorable pressure gradient flow is mainly due to the higher mean velocity of the fluid. For the same reason, the thermally fully developed region is much shorter than that of a pure electroosmotic flow with the adverse pressure gradient, simply because the fluid has more time to exchange

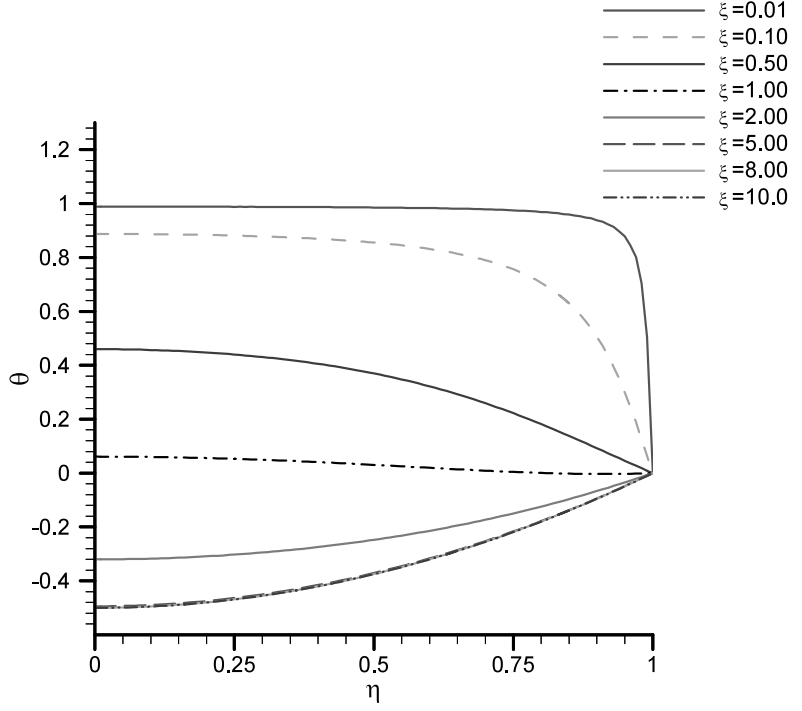

(a)

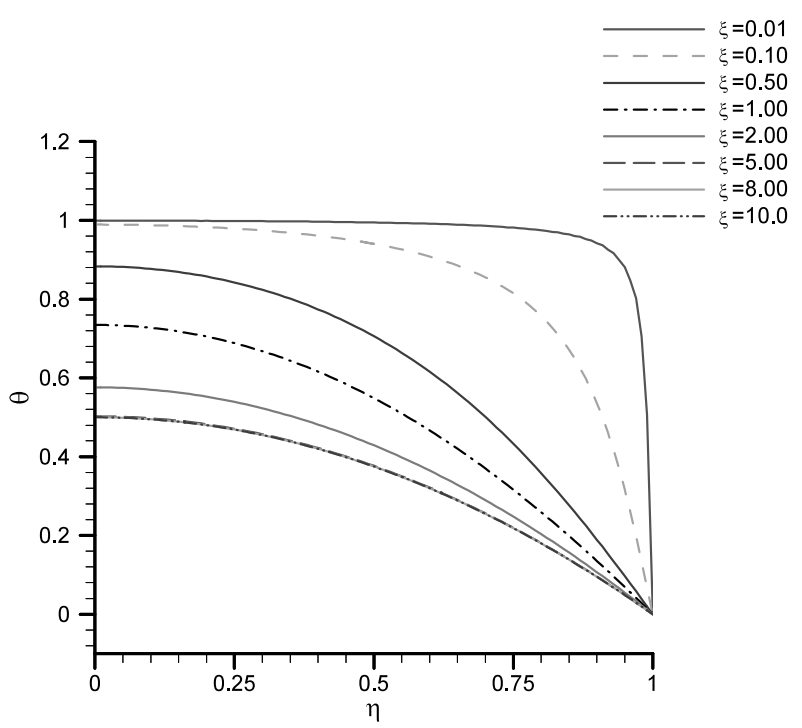

(b)

Fig. 2 Temperature distribution in mixed electroosmotic and pressure driven flow for $\Omega=0$ at different longitudinal locations at $P e_{T}=1$ for (a) $G=-1$ and (b) $G=+1$

heat with the channel walls.

In all cases presented above (Figs. 2-4), the fully developed temperature in the dimensional form, $T_{f d}=$ $\left(T_{e}-T_{S}\right) \theta_{f d}+T_{S}$, at any lateral location is the same for each particular value of $|G|$. To be specific, according to Figs. 2-4, the normalized centerline temperature in the fully developed region eventually reaches $\theta_{f d, C}=0.5 G$, in all cases. This fully developed temperature can be easily explained by substituting $\xi \rightarrow \infty$ into Eq. (12).

Figure 5 shows the local Nusselt number distribution along the channel for two cases $(\Omega=-1$ and 1) presented in Figs. 2-4, for normalized source term $G=-1$ and 1. Due to the identical nature of the local Nusselt number after reaching the fully developed condition, they have 


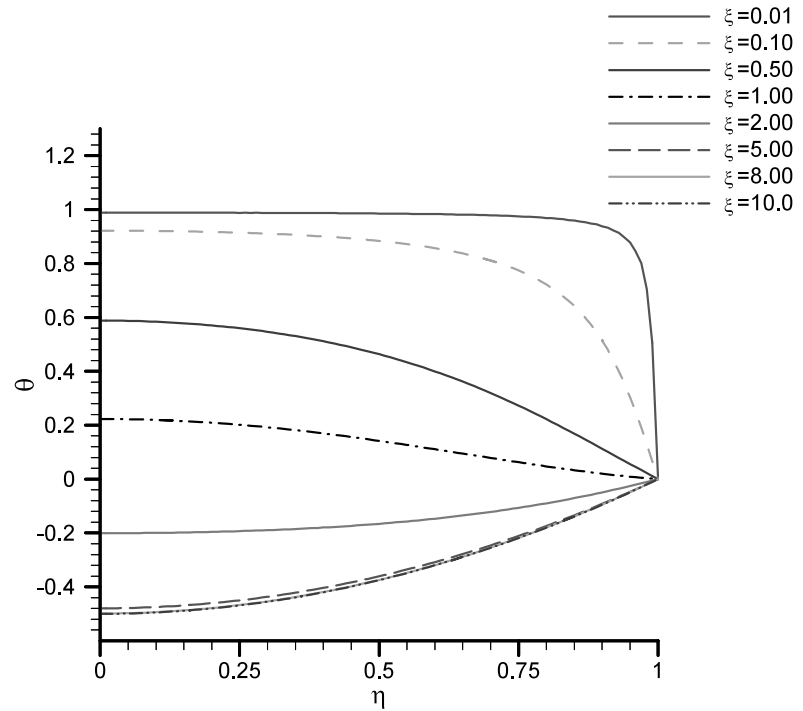

(a)

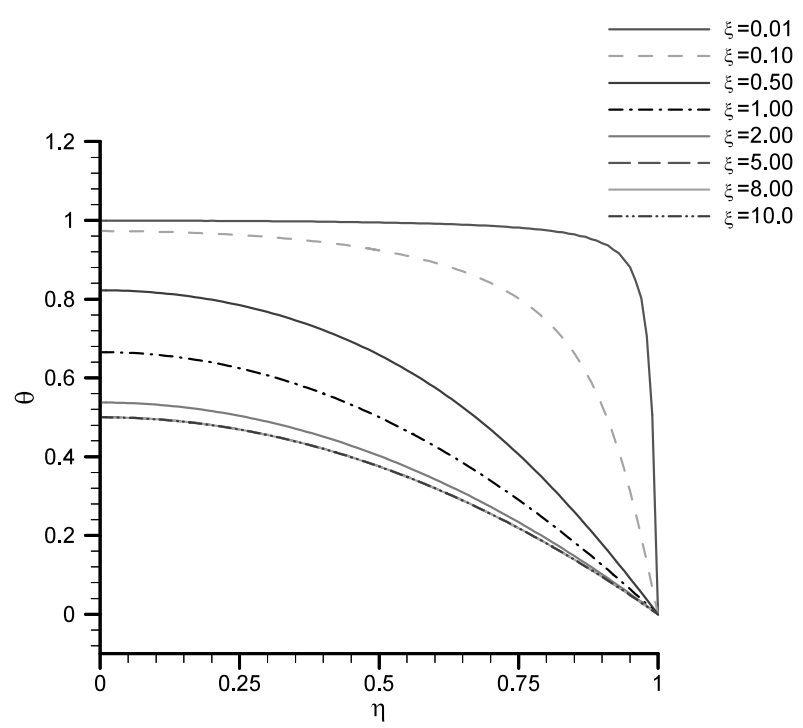

(b)

Fig. 3 Temperature distribution in mixed electroosmotic and pressure driven flow for $\Omega=+1$ at different longitudinal locations at $P e_{T}=1$ for (a) $G=-1$ and (b) $G=+1$

been presented up to $\xi=100$. The local Nusselt number abruptly decays to reach a flat value within twenty characteristic lengths $(\xi=20$ or $x=20 D)$. The rationale for this abrupt decay is that a thermal boundary layer develops along the direction of the flow. The spike in the local Nusselt number distribution for $G=-1$ indicates the location along the channel where the mean temperature approaches the surface temperature. For negative normalized generation $(G<0)$, the fluid temperature $(T)$ increases due to both the Joule heating and the surface thermal condition, until it exceeds the surface temperature. It is clear that the fully developed Nusselt number approaches the same value as long as the magnitude of $G$ is identical. For positive normalized generation $(G>0)$, the fluid tempera-

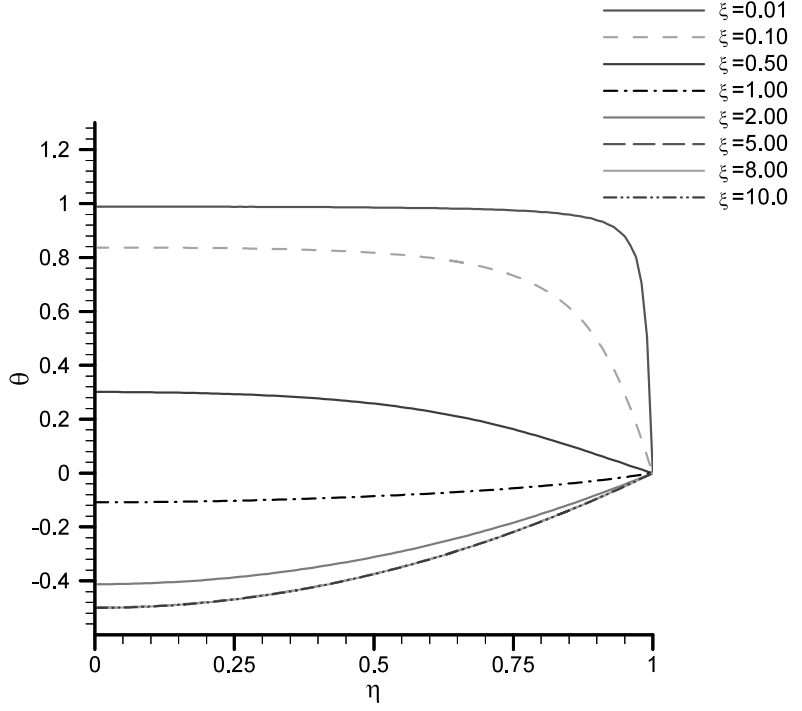

(a)

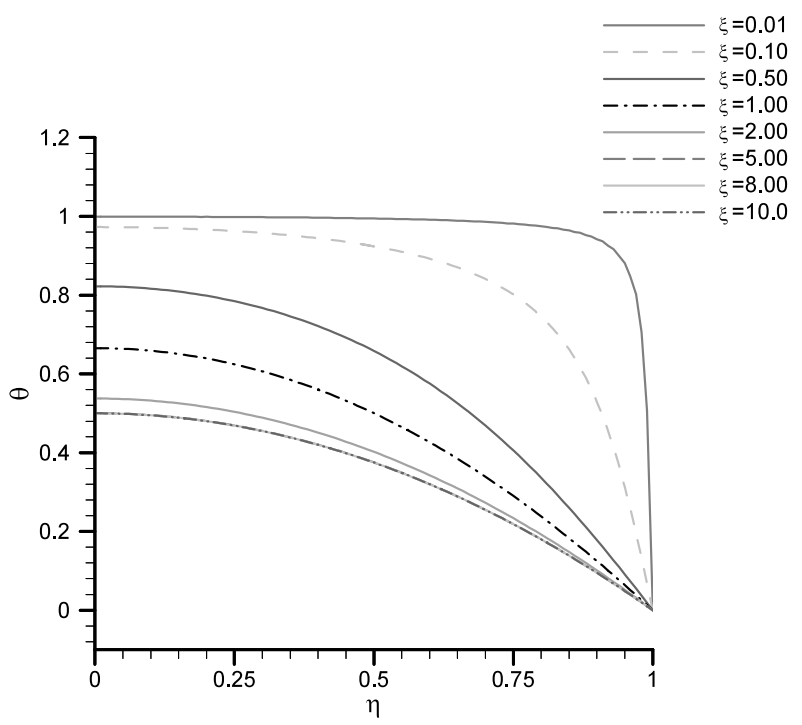

(b)

Fig. 4 Temperature distribution in mixed electroosmotic and pressure driven flow for $\Omega=-1$ at different longitudinal locations at $P e_{T}=1$ for (a) $G=-1$ and (b) $G=+1$

ture $(T)$ decreases constantly in order to satisfy the lower wall surface temperature boundary, whereas heat generation exists inside the channel. It turns out that the entrance length is the same for the same absolute value of normalized heat generation, $G$, due to energy balance between the amount of heat generation and heat rejection, while the entrance length gets longer as the pressure gradient is increased.

For each case, we also studied the dependence of the thermal Peclet number based on the HelmholtzSmoluchowski velocity. For a particular flow, the fully developed Nusselt number is independent of the Peclet number, and it does not depend on the magnitude of the source term. The fully developed Nusselt numbers for dif- 


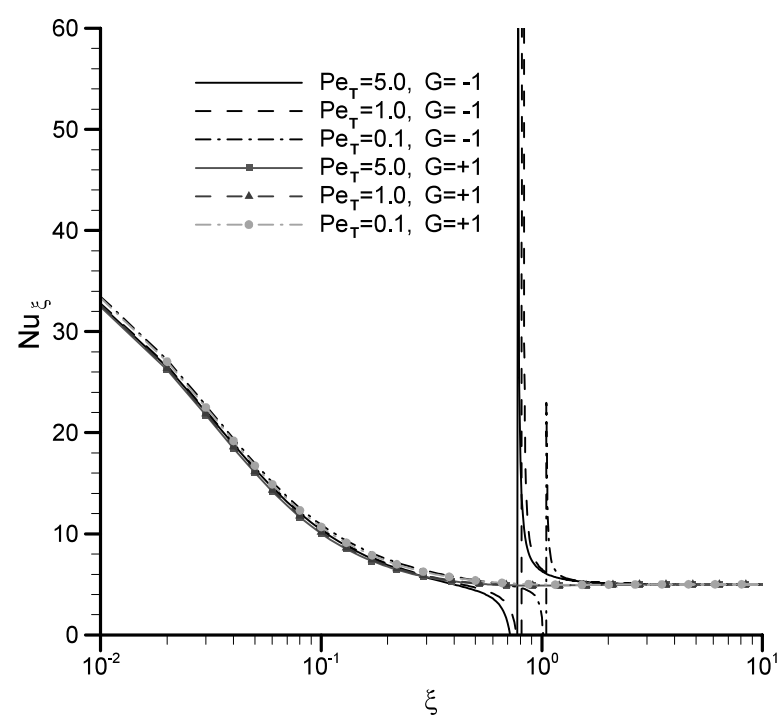

(a)

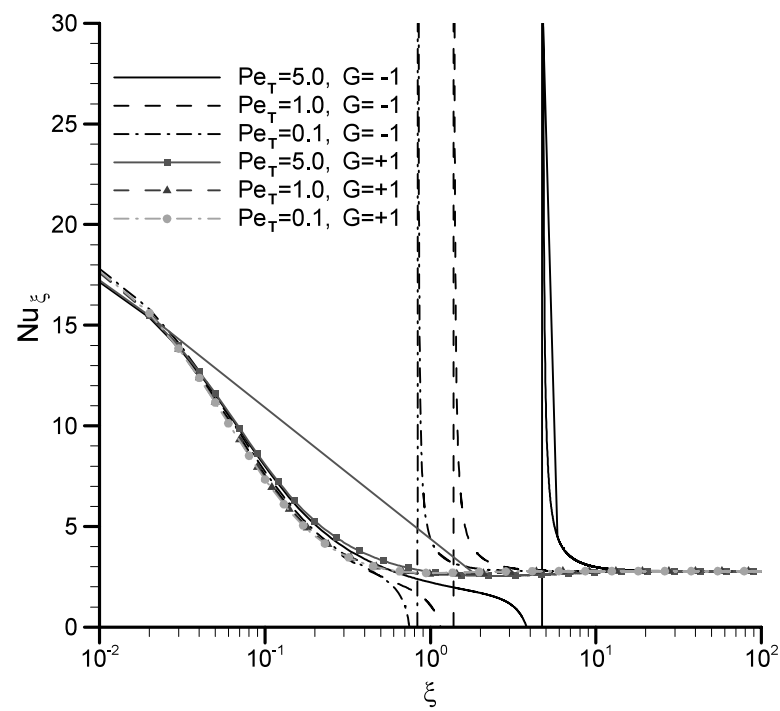

(b)

Fig. 5 Local Nusselt number distribution along channel for different Joule heating values at different Peclet numbers for (a) $\Omega=-1$ and (b) $\Omega=+1$

ferent source terms are presented in Table 2. Here, our Nusselt number values are computed based on their hydraulic diameter $\left(N u_{D_{H}}=4 N u_{D}\right)$. For both positive and negative source terms with the same magnitudes considered in this study $(G=-1$ and 1$)$, the Nusselt number in the fully developed region becomes the same. In pure electroosmotic flow, the Nusselt number reaches a value of 12.0 in the fully developed region, which agrees with other researchers ${ }^{(16)}$. For cases without Joule heating the heat transfer characteristics of pure electroosmotic flow are identical to the classical isothermal heat transfer in a slug flow, which the fully developed Nusselt number is $9.868^{(17)}$. In the fully developed region, the Nusselt number for the electroosmotic flow is significantly higher than
Table 2 Comparison of Nusselt numbers based on hydraulic diameter $\left(N u_{D_{H}}\right)$ in fully developed region compared with existing literature

\begin{tabular}{|c|c|c|c|c|}
\cline { 2 - 5 } \multicolumn{1}{c|}{} & \multicolumn{2}{c|}{$\mathrm{G}= \pm 1$} & \multicolumn{2}{c|}{$\mathrm{G}=0$} \\
\cline { 2 - 5 } \multicolumn{1}{c|}{} & This Study & Reference & This Study & Reference \\
\hline Mixed Flow $(\Omega=-1.0)$ & 20.00 & - & 18.90 & - \\
\hline Electroosmotic flow & 12.00 & $12.00^{(16)}$ & 9.87 & $9.87^{(17)}$ \\
\hline Mixed Flow $(\Omega=+1.0)$ & 11.11 & - & 8.80 & - \\
\hline Mixed Flow $(\Omega=+5.0)$ & 10.40 & - & 7.98 & - \\
\hline Poiseuille flow & 10.00 & - & 7.59 & $7.59^{(10)}$ \\
\hline
\end{tabular}

that of the Poiseuille flow. The higher heat transfer characteristics in a pure electroosmotic flow can be attributed to the plug-like uniform velocity. In pure Poiseuille flows, the fully developed Nusselt number also compares with that in existing literature ${ }^{(10)}$ for identical geometric conditions. In the fully developed region our analytical results exactly match the other existing studies. Note that the heat flux at the wall is the same for all cases in the fully developed region, and it can be explained by the temperature distribution in Figs. 2 -4. This implies that the normalized bulk mean temperature gets higher as the pressure gradient increases, which solely depends on the normalized velocity distribution across the channel. In other words, a higher flow rate leads to less heat transfer.

\section{Conclusions}

We obtained analytical solutions for the heat transfer characteristics of mixed electroosmotic-pressure driven flows in two-dimensional straight microchannels for constant zeta potentials, buffer concentrations, and external electric fields. This analysis takes care of the interaction among advective, viscous, and Joule heating terms to obtain the temperature distribution within the fluid. Our analysis resulted in the following:

1. For an isothermal channel surface condition before it reaches an identical profile, the local fluid temperature in dimensional form $(T)$ decreases for a positive source term $(G>0)$ and increases for a negative source term $(G<0)$ along the channel.

2. For a particular flow type (in the fully developed region), the normalized heat transfer coefficient reaches the same value for both the positive and negative source terms.

3. The Nusselt number of the mixed electroosmotic and pressure driven flow is smaller than that of the pure electroosmotic flow for favorable pressure gradients.

4. Electroosmotic flow with adverse pressure gradient provides the best heat transfer performance.

5. The temperature profile in the fully developed region is independent of the Peclet number and pressure gradient. 
6. In the mixed electroosmotic-pressure driven flows, the thermal entrance length increases with the imposed pressure gradient.

7. The entrance length is the same for the same magnitude of normalized heat generation, $G$.

\section{Acknowledgements}

This work has been partially funded by Washington State University Office of Research and partially by the National Science Foundation (CTS 0300802). The authors would like to thank Prof. Hong-Ming Yin for his valuable suggestions.

\section{References}

( 1 ) Chen, C.H. and Santiago, J.G., A Planar Electroosmotic Micropump, J. Microelectromechanical Systems, Vol.11 (2002), pp.672-683.

( 2 ) Reuss, F.F., Charge-Induced Flow, Proceedings of the Imperial Society of Naturalists of Moscow, Vol.3 (1809), pp.327-344.

( 3 ) Burgreen, D. and Nakache, F.R., Electrokinetic Flow in Ultrafine Capillary Silts, J. Physical Chemistry, Vol.68 (1964), pp.1084-1091.

( 4 ) Rice, C.L. and Whitehead, R., Electrokinetic Flow in a Narrow Cylindrical Capillary, J. Physical Chemistry, Vol.69 (1965), pp.4017-4024.

( 5 ) Dutta, P. and Beskok, A., Analytical Solution of Combined Electroosmotic/Pressure Driven Flows in TwoDimensional Straight Channels: Finite Debye Layer Effects, Analytical Chemistry, Vol.73 (2001), pp.1979_ 1986.

( 6 ) Soderman, O. and Jonsson, B., Electro-Osmosis: Velocity Profiles in Different Geometries with Both Temporal and Spatial Resolution, J. Chern. Phys., Vol.105 (1996), pp.10300-10311.
( 7 ) Wong, P.K., Chen, C.Y., Wang, T.H. and Ho, C.M., An AC Electroosmotic Processor for Biomolecules, Proc. of 12th International Conference on Solid State Sensors, Actuators and Microsystems, Vol.1 (2003), pp.20-23.

( 8 ) Zeng, S., Chen, C.H., Mikkelsen, J.C., Jr. and Santiago, J.G., Fabrication and Characterization of Electroosmotic Micropumps, Sensors and Actuators B, Vol.79 (2001), pp.107-114.

( 9 ) Maynes, D. and Webb, B.W., Fully-Developed Thermal Transport in Combined Pressure and ElectroOsmotically Driven Flow in Microchannels, ASME J. Heat Transfer, Vol.125 (2003), pp.889-895.

(10) Lahjomri, J. and Oubarra, A., Analytical Solution of the Graetz Problem with Axial Conduction, ASME J. Heat Transfer, Vol.121 (1999), pp.1078-1083.

(11) Hunter, R.J., Zeta Potential in Colloid Science: Principles and Applications, First ed., (1981), Academic Press Inc, New York.

(12) Probstein, R.F., Physiochemical Hydrodynamics, Second ed., (1994), Wiley and Sons Inc., New York.

(13) Arfken, G., Mathematical Methods for Physicists, Third ed., (1985), Academic Press, Florida.

(14) Gobie, W.A. and Ivory, C.F., Thermal Model of Capillary Electrophoresis and a Method of Counteracting Thermal Band Broadening, J. Chromatography, Vol.516 (1990), pp.191-210.

(15) Burgi, D.S., Salomon, K. and Chien, R.L., Methods for Calculating the Internal Temperature of Capillary Columns during Capillary Electrophoresis, J. Liquid Chromatography, Vol.14 (1991), pp.847-867.

(16) Maynes, D. and Webb, B.W., Fully Developed ElectroOsmotic Heat Transfer in Microchannels, Int. J. Heat and Mass Transfer, Vol.46 (2003), pp.1359-1369.

(17) Burmeister, L.C., Convective Heat Transfer, Second ed., (1993), Wiley and Sons, New York. 